\title{
Dentofacial injuries and domestic abuse
}

\author{
Abstracted from
}

Coulthard P, Yong SL, Adamson L et al.

Domestic violence screening and intervention programmes for adults with dental or facial injury. Cochrane Database Syst Rev 2010; (12): CD004486.

Address for correspondence: Luisa Fernandez Mauleffinch, Review Group Co-ordinator, Cochrane Oral Health Group, MANDEC, School of Dentistry, University of Manchester, Higher Cambridge Street, Manchester, M15 6FH, UK.

E-mail: luisa.fernandez@manchester.ac.uk

\section{Question: Are screening and intervention programmes for dental and facial injuries effective at reducing or preventing domestic violence in adults?}

Data sources Ovid, Medline, EMBASE, PsycINFO, CINAHL, LILACS, CENTRAL, bibliographies of identified RCTs, Current Controlled Trials, domestic violence organisations, handsearching of a number of articles. Study selection This is an update of a review reported in 2004. Randomised controlled trials in any language that included adults (persons > 16 years of age) presenting with facial or dental injuries to dental or healthcare settings. Eligible interventions were: referral to support services or screening for domestic violence. Eligible outcomes were: frequency of physical assault by self-report, severity of injury by self-report and proportion of victims of domestic violence detected by self-report.

Data extraction and synthesis Two reviewers screened potential reports and would have data extracted had suitable RCTs been included.

Results No RCTs met the inclusion criteria.

Conclusions There is also no evidence from RCTs to suggest that screening for domestic violence in adults with dental or facial injury in any health setting is beneficial or harmful.
This paper is based on a Cochrane Review published in the Cochrane Library 2010, issue 12 (see www.thecochranelibrary.com for information). Cochrane Reviews are regularly updated as new evidence emerges and in response to feedback, and the Cochrane Library should be consulted for the most recent version of the review.

\section{Commentary}

This article deals with the difficult issue of domestic abuse (DA). This issue may be seen to lie outside the remit of the general practitioner, and is an issue that many may feel uncomfortable raising with patients, yet it is an issue which will present to them during their practising life. One in four women will experience DA at some point in their lives and we know that a facial bruise in a female patient is 32 times more likely to be due to DA than any other cause. So although abuse may take different forms, many victims will suffer injuries to the mouth, head or neck, putting the general practitioner in a very good position to spot the signs.

This review looked for evidence of the effectiveness of interventions that screened patients for domestic abuse or referred them on to specialist agencies for support. Outcome measures were the frequency and severity of injury and the proportion of victims detected by self-report. Unfortunately no articles met these criteria so the authors were unable to draw a firm conclusion from the available information. Outcome measures in this area are always going to be difficult to assess and self-report is somewhat unreliable, however, it is unfortunate given the prevalence of domestic abuse that more good quality work has not been done in this area, as there is clearly a need for it.

So why do we stick our heads in the sand about it in both a practical and research sense? One reason is that we believe that victims do not wish to be asked about abuse and will be offended, yet research has shown that if a female patient presents to the dentist following abuse most wish to be given the opportunity to talk about it. Another reason is that we don't know what to do about it and worry, quite reasonably, about doing more harm than good. However, as health professionals who have regular contact with their patients, dentists are in a good position to ask about abuse, to provide support, reassurance and validation, to document the signs of abuse for any future court proceedings and to provide contact details of DA organisations (The AVDR approach). There is some evidence from an RCT that training in this less intensive approach can be effective in terms of increasing the knowledge, attitudes and behaviours of dentists in relation to domestic abuse, and according to the Theory of Planned Behaviour this should translate into an increase in detection of victims, which was one of the desired outcomes of this review.

Christine A Goodall

Department of Oral Surgery, Glasgow University Dental School, Glasgow, Scotland, UK

Evidence-Based Dentistry (2012) 13, 86. doi:10.1038/sj.ebd.6400879 